Historic, Archive Document

Do not assume content reflects current scientific knowledge, policies, or practices. 


\section{WHOLESALE TRADE CATALOGUE}

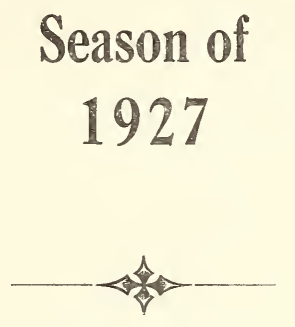

for Nurserymen, Florists, and Landscape Architects only.

SPEGIAL SOUTHERN LANDSCAPE PLANTS

Fungle Gardens AVERY ISLAND, LOUISIANA. 


\section{INTRODUCTORY}

WE OFFER THE FOLLOWING Nursery Stock for the season of 1927, and shall be very glad indeed to have you read same carefully, advising us if we may serve you, as we have a first class stock of plants, shrubbery, etc. It would be good policy to place your order early for your wants, as there is likely to be a shortage in nursery stock in some varieties.

WE MAKE A SPECIALTY of plants in size and variety adapted for SOUTHERN PLANTING AND LANDSCAPE WORK. Our stock of LARGE plants is the FINEST IN THE SOUTH.

\section{SYMBOLS}

B. \& B. Signifies BALLED and BURLAPPED (taken up with ball of dirt and wrapped in burlap.)

N. B. Signifies NOT BALLED (taken up bare rooted.)
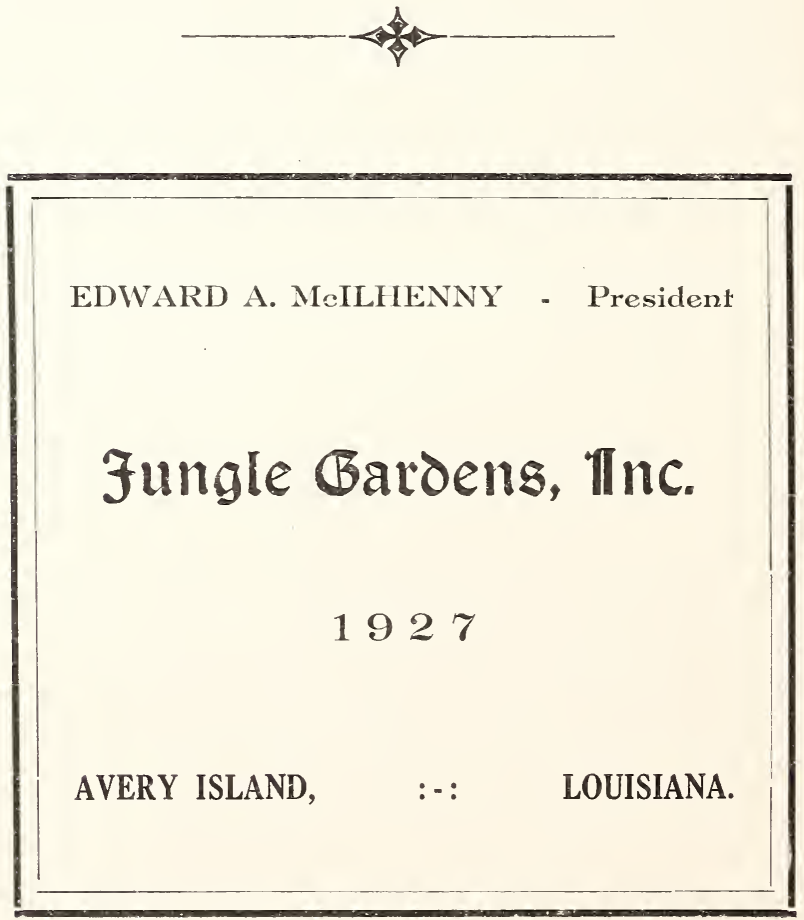


\section{TERMS OF BUSINESS}

Location.-General Office and Nursery at Avery Island, Iberia Parish, Louisiana, on the Southern Pacific Railway, and hard-surface highway through Nursery, eight miles south of New Iberia, Louisiana.

Telegraph Address is New Iberia, Louisiana.

Long Distance Telephone office in our main office.

Our Freight Station is Salt Mine, Louisiana.

Invitations to Visitors.-We take pleasure in showing our stock to persons wishing to purchase; if notified in time, we will meet visitors at the station on arrival.

Terms.-Cash with order, if for immediate shipment. Orders for trees to be held for some weeks after the shipping season opens should also be accompanied by full payment. On orders booked in advance of the shipping season, 25 per cent down, with the balance due when the shipping season opens. We do not care to ship C. O. D.

Remittances.-To secure safety and prompt acceptance, remittances should be made by Bank Draft, Express or Post-Office Money Order, Registered Letter, or Prepaid Express.

Method of Shipment.-Shipping directions should be plainly written, and we urge our customers, in all cases, to use our order sheets. We shall be pleased to forward additional ones upon application. Kindly indicate whether shipment is to be made by freight, express or mail. When method of shipment is left to us, or when no directions are given, we will ship according to our best judgment, by such means as we believe to be in the best interests of our customers.

Transportation at Purchaser's Risk and Expense.All transportation charges are to be paid by the purchaser, and our responsibility ceases upon delivery to forwarding companies; claims for losses or damages must be made upon the latter. We will, however, start a tracer for delayed shipment, if notified, and use every means at our command to secure prompt delivery, or recovery in case of damage or loss.

Time of Shipment.-Unless instructed otherwise, orders during the shipping season will be forwarded as soon after their receipt as possible, and orders booked in advance will be shipped as soon as may be after the shipping season opens.

Shipping and Planting Season.-November to March for all trees and shrubs grown in the open field; balled plants, bamboos, grasses and pot-grown plants may be shipped at any time. Prices in this catalogue cover the shipping season of 1927.

Selecting Varieties suited to locality, is of the first importance, and can often be more advantageously done by ourselves than by the purchasers. We are always glad to aid our customers in their selections, when so desired, and will, upon request, cheerfully furnish additional information in regard to the adaptability or desirability of particular varieties.

Labels attached with brass wire to everything sent out.

We Guarantee all stock sent out to be well rooted, well grown, true to name, properly packed, and shipped according to instructions. Our liability under the foregoing guaranty is limited in amount to the original price received.

Claims.-If, by any possibility, errors should occur, they will be promptly rectified, if claim is made within ten days after the receipt of the goods. 


\section{DECIDUOUS SHADE TREES}

\section{SIZE}

Class Per 10

ACER (Maple)

A. dasycarpum (Southern Maple) or (Soft Maple) 6-8 feet

A. pseudoplatanus (Sycamore Maple)

$6-8$ feet
AILANTHUS $\mathrm{NB} \$ 12.50$

\section{A. grandulosa (Tree-of-heaven)} 6-8 feet

ALBIZZIA

A. julibrissin (Mimosa)

3-4 feet

$5-6$ feet

8-10 feet

NB $\quad 15.00$

ALEURITES

A. fordi (Tung-oil Tree)

3-4 feet

6-7 feet

8 feet and up

\section{BACCHARIS}

\section{B. halmifolia} 6-8 feet

$\begin{array}{lr}\text { NB } & 6.50 \\ \text { NB } & 12.50 \\ \text { NB } & 30.00\end{array}$

\section{CATALPA}

C. speciosa (Western Catalpa) 6-8 feet

CELTIS (Hackberry)

C. occidentalis

CERCIS (Redbud or American Judas Tree)

C. canadensis (American Redbud) 4-5 feet

6-7 feet

CORNUS (Dogwood)

C. florida (Flowering Dogwood) $11 / 2$ feet 4-5 feet

FRAXINUS' (Ash)

F. americana alba (White Ash) 6-8 feet

KOELREUTERIA

K. paniculata (Goldenrain-tree)

$\mathrm{K}$. bipinnata

3-5 feet

6-8 feet

LIQUIDAMBAR

L. styraciflua (Sweetgum)

5- 6 feet

6-7 feet

\section{MANIHOT}

M. carthaginensis (Yuquilla Tree)

$$
\text { 4-5 feet }
$$

6-7 feet

NB $\quad 7.50$

NB $\quad 10.00$

\section{MELIA}

M. umbraculiformis (Texas Umbrella-tree)

$$
\text { 3-4 feet }
$$
5-6 feet

NYSSA (Tupelo)

N. sylvatica 5-6 feet 6-8 feet 
POPULUS (Poplar)

P. alba nivea (Silver Poplar)

P. deltoides (Carolina Poplar)

6-8 feet

P. nigra italica (Lombardy Poplar)

4-5 feet - NB 3.50

8-9 feet _ NB 20.00

10 feet ___ B \& B 40.00

P. simoni fastigiata (Narrow Simon Poplar)

6-7 feet - NB 12.50

8-9 feet - NB 20.00

QUERCUS (Oak)

Q. nigra (Water Oak)

6 feet, $1 \frac{1 / 2}{2}$ inch caliper______B \& B 27.50

8 feet, 4 inch caliper

Q. palustris (Pin Oak)

$\begin{array}{llll}6-7 \text { feet } & \text { B \& B } & 27.50 \\ 7-8 \text { feet } & \text { B \& B } & 40.00\end{array}$

ROBINIA (White Locust)

R. pseudoacacia 4-5 feet

8-9 feet

NB $\quad 10.00$

NB $\quad 20.00$

SALIX (Willow)

(Common Willow)

5-6 feet - NB $\quad 5.00$

6-8 feet - NB 10.00

S. elegantissima (Thurlow Weeping Willow)

4-5 feet _._ NB 6.50

6-8 feet _-_ NB 12.50

SAMBUCUS (Elder)

S. canadensis laciniata (Cutleaf American Elder) 2-3 feet

NB 8.00

\section{SAPINDUS}

S. indica (Indian Soap Tree)

4-5 feet

NB $\quad 3.50$

\section{SAPIUM}

S. sebiferum (Chinese Tallowtree)

4-5 feet

7-8 feet, 1 inch caliper

NB $\quad 3.50$

NB $\quad 15.00$

\section{SASSAFRAS}

S. variifolium

6-8 feet

NB

\section{SPONDIAS}

S. lutea (Yellow Mombin)

S. axillaris

6-8 feet

NB $\quad 15.00$

TAXODIUM

T. distichum (Native Cypress)

4-5 feet

5-6 feet

\section{ULMUS' (Elm)}

U. americana (American Elm) 4-5 feet 8-10 feet

U. parviflora (Weeping Chinese Elm) 3-4 feet

U. pumila (Upright Chinese Elm) 3-4 feet 


\section{DECIDUOUS PLANTS}

SIZE

Class

Per 10

ACACIA

A. farnesiana (Sweet Acacia)

3-4 feet

NB $\quad 6.00$

5-6 feet

9.50

ARALIA

A. pentaphylla, Acanthopanax pentaphyllum

A. ${ }^{3-4}$ feet spinosa (Devils-walkingstick)

3-6 feet
CALLICARPA (Beautyberry) or (Pigeonberry)

C. americana

C. longifolia

2-3 feet

3-4 feet

NB $\quad 4.50$

NB $\quad 6.50$

CASSIA

C. corymbosa (floribunda) (Flowery Senna)

(Small Yellow)

C. floribunda gigantae (Large Yellow)

3-4 feet

6.00

4-6 feet

$\mathrm{NB}$

7.50

CYDONIA (Quince)

C. japonica (Flowering Quince)

2-3 feet

3-4 feet

2-3 feet

$\begin{array}{rr}\text { NB } & 4.50 \\ \text { NB } & 6.00 \\ \text { B \& B } & 10.00\end{array}$

3-4 feet

B \& B 12.50

DEUTZIA

D. Scabra (Fuzzy Deutzia) or (Pride of Rochester)

4-5 feet

2-3 feet ___ B \& B 10.00

4-5 feet

DIOSPYROS (Persimmon)

Native and Jananese

2-3 feet

4-5 feet

NB $\quad 12.50$

NB $17.5 \circ$

FORSYTHIA

F. viridissima (Greenstem Forsythia)

2-3 feet NB

$3-4$ feet $\quad$ NB 5.00

2-3 feet ____________ B \& B 10.00

3-4 feet

HIBISCUS (Hibiscus, Rosemallow)

H. moscheutos (Mixed colors)

2 year old roots

NB $\quad 6.00$

H. syriacus (Althea Rose of Sharon)

Dou. White and Dou. Pink

2-3 feet

-- NB 4.00

4-5 feet

$2-3$ feet ___ $B$ B \& B

4-5 feet

B \& B 15.00

HYDRANGEA

(French Hybrids)

2 year old plants.

NB $\quad 10.00$

HYPERICUM (St. Johnswort)

H. moserianum (Goldflower)

$11 / 2-2$ feet

$2-21 / 2$ feet

NB

NB

3.50

4.50

LAGERSTROEMIA (Crapemyrtle)

L. indica alba (White Crapemyrtle)

L. indica purpurea (Purple Crapemyrtle)

L. indica rosea (Pink Crapemyrtle)

L. indica (Watermelon-red Crapemyrtle) 2-3 feet

3-4 feet NB

2-3 feet NB

3-4 feet 
PARKINSONIA

P. aculeata

3-4 feet

NB $\quad 6.00$

5-6 feet

12.50

PLUMBAGO

P. capensis (Cape Plumbago)

$1 \frac{1 / 2}{2}$ feet

$21 / 2-3$ feet

NB $\quad 6.00$

NB $\quad 7.50$

PUNICA (Pomegranate)

P. legrellei (Flowering Pomegranate)

$P$. nana racemosa (Flowering Pomegranate) 3-4 feet

4-5 feet

RHUS (Sumac)

R. glabra (Smooth Sumac)

4-5 feet

R. typhina laciniata (Shredded Sumac) or (Fernleaf Sumac) 3-4 feet

NB

\section{SPIRAEA}

S. bumalda (Anthony Waterer Spirea) (Crimson) 15-18 inches

S. billiardi (Billiardi Spirea) (Pink)

S. douglasi (Douglas Spirea)

S. prunifolia (Bridal Wreath)

S. vanhouttei (Vanhoutte Spirea)

$\begin{array}{rrr}2-3 \text { feet } & \text { NB } & 3.50 \\ 3-4 \text { feet } & \text { NB } & 4.50 \\ 2-3 \text { feet } & \text { B \& } & 7.50 \\ 3-4 \text { feet } & \text { B } & 10.00 \\ \text { reevesiana (Reeves Spirea) } & & \end{array}$

S. reevesiana (Reeves Spirea)

S. thunbergi (Thunberg Spirea)

$15-18$ inches

NB $\quad 3.50$

TAMARIX

T. gallica (French Tamarix) (Salt Cedar)

T. odessana (Odessa Tamarix)

3-4 feet

$\begin{array}{ll}\text { NB } & 2.50 \\ \text { NB } & 4.00\end{array}$

5-6 feet

VITEX (Chaste-tree)

V. agnus-castus (Lilac Chaste-tree)

V. agnus-castus alba (White Chaste-tree)

V. negundo (Blue Chaste-tree)

V. incisa (Cutleaf Chaste-tree)
2-3 feet
NB
4-5 feet $\mathrm{NB}$
3-4 feet
$\mathrm{B} \& \mathrm{~B}$
2.00
5.00
10.00
5-6 feet
B \& B
19.00

WEIGELIA (Diervilla)

W. rosea (florida) (Pink Weigelia) 2-3 feet

3-4 feet

NB

2-3 feet

$B$ \& B

3-4 feet

10.00

12.50

\section{EVERGREEN PLANTS}

ABELIA

A. grandiflora (Glossy Abelia)

$\begin{array}{lrr}2-3 \text { feet } & \text { NB } & 4.50 \\ 3-4 \text { feet } & \text { NB } & 7.00 \\ 2-3 \text { feet } & \text { B \& B } & 14.00 \\ 3-4 \text { feet } & 20.00\end{array}$

AZALEA

A. indica formosa $1-1 \frac{1}{2}$ feet 
BUDDLEIA (Butterflybush)

B. asiatica (Winter blooming-White)

B. officinalis (Winter blooming-Lilac)

$$
\text { 3-5 feet B \& B }
$$

B. variabilis In variety (Summer blooming)

3-4 feet

B \& 17.50

BUXUS (Box)

B. sempervirens (Common Box)

9-12 inches

$1 \frac{1 / 2}{2} 2$ feet

(Common Creole Box)

9-12 inches

$1 \frac{1 / 2}{2} 2$ feet

\section{CAMELLIA}

C. japonica (Common Camellia)

2-3 feet

B \& B

15.00

COCCULIS

C. laurifolia

3-4 feet

B \& B

30.00

DURANTA

D. plumeri 3-4 feet

B \& B

20.00

\section{ELAEAGNUS}

E. pungens reflexa (Bronze Elaeagnus)

2-3 feet

B \& B

3-5 feet.

B \& B

15.00

20.00

ERYTHRINA

E. crista-galli

E. herbacea

3-4 feet

B \& B

25.00

ESCALLONIA

E. berteriana

3-4 feet

B \& B

20.00

FATSIA

F. papyrifera, Tetrapanax papyriferum

2-3 feet

3-6 feet

B \& B 12.00

17.50

GARDENIA

G. florida (Cape-jasmine)

1-2 feet

2-3 feet

1-2 feet

2-3 feet

B \& B

ILEX (Holly)

I. vomitora (Yaupon)

$1 \frac{1 / 2}{2}-2$ feet

B \& B

3-4 feet

B \& B

5-6 feet

B \& B

JASMINUM (Jasmine)

(Large leaf bush Jasmine-Yellow)

3-4 feet B \& B

(Small leaf bush Jasmine-Yellow)

3-4 feet B \& B

J. nudiflorum

2-3 feet B \& B

J. primulinum (Primrose Jasmine)

2-3 feet

$\mathrm{B} \& \mathrm{~B}$

6-9 feet

B \& B

2.00

3.00

6.00

10.00

12.00

17.50

25.00

14.00

15.00

12.50

12.00

25.00

LIGUSTRUM (Privet)

L. lucidum (Glossy Privet)

4-5 feet

6-7 feet

B \& B

B \& B

L. Iucidum superbum-Grafted $4 \frac{1 / 2-5}{\text { feet }}$ 
LIGUSTRUM (Privet)

L. gracilis (Long Branched Waxleaf)

L. iwata variegata (Low Bushy)

L. nobilis (Tall Upright)

$\begin{array}{lll}3-4 \text { feet } & \text { B \& B } & 12.00 \\ 4-5 \text { feet } & \text { B \& B } & 15.00\end{array}$

MALVAVISCUS

M. grandiflora (Turk's Cap) $2-3$ feet B \& B 12.50 4-5 feet B \& B 20.00

\section{MICHELIA}

M. fuscata (Magnolia fuscata) or (Banana-shrub) 1-2 feet

B \& B 12.50

2-3 feet

B \& B 20.00

MYRICA (Bayberry, Waxmyrtle)

M. cerifera (Southern Waxmyrtle)

3-4 feet

5-6 feet

B \& B 15.00

B \& B 22.50

MYRTUS (Myrtle)

M. communis (True Myrtle)

1-2 feet

2-3 feet

B \& B 12.50

B \& B 25.00

NANDINA

N. domestica

2 feet

3-4 feet

B \& B

B \& $B$

15.00

20.00

NERIUM (Oleander)

(Cream)

(Double Pink)

(Single Pink)

(Red)

(White)

2-3 feet

4-5 feet

B \& B 12.50

5-6 feet

B \& B 22.50

B \& B $\quad 35.00$

OSMANTHUS

O. aquifolium (Holly Olive)

1-2 feet

B \& B 12.50

2-3 feet

B \& B 17.50

o. fragrans (Sweet Olive)

2-3 feet

B \& B $\quad 17.50$

PHOTINIA

P. serrulata

4-5 feet

5-6 feet

B \& B $\quad 30.00$

B \& B $\quad 50.00$

\section{PITTOSPORUM}

P. tobira

1 to 2 feet spread

B \& B 10.00

2 to 3 feet spread

3 to 4 feet spread

B \& B 17.50

P. to B \& B

25.00

1 to 2 feet spread

2 to 3 feet spread

17.50

3 to 4 feet spread

B \& B

B \& B

25.00

\section{PRUNUS}

P. caroliniana (Carolina Cherry-laurel)

2-3 feet .

3-4 feet

$B \& B$

15.00

20.00

TECOMARIA

T. capensis (Cape Honeysuckle) 2-3 feet B \& B $\quad 17.50$

\section{VIBURNUM}

V. tinus (Laurustinus)

V. suspensum

2-3 feet

3-4 feet

B \& B

B \& B $\quad 15.00$ 


\section{EVERGREEN SHADE TREES}

\section{SIZE}

Class Per 10

CINNAMOMUM

C. camphora (Camphor-tree)

$\begin{array}{lll}3-4 \text { feet } & \text { B \& B } & 20.00 \\ 4-5 \text { feet } & \text { B \& B } & 25.00 \\ 6-7 \text { feet } & \text { B \& B } & 40.00 \\ 7-8 \text { feet } & \text { B \& B } & 50.00\end{array}$

ERIOBOTRYA (Loquat)

E. japonica (Loquat)

$\begin{array}{lll}2-3 \text { feet } & \text { B \& B } & 14.00 \\ 3-4 \text { feet } & \text { B \& B } & 20.00 \\ 5-6 \text { feet } & \text { B \& B } & 45.00\end{array}$

ILEX (Holly)

I. opaca (American Holly)

$3-4$ feet

B \& B 15.00

4-5 feet

6-7 feet

B \& B 25.00

MAGNOLIA

M. glauca (Sweet Bay)

$3-4$ feet.

$B$ \& B

4-5 feet

B \& B 25.00

5-6 feet

B \& B $\quad 35.00$

MAGNOLIA

M. grandiflora (Southern Magnolia)

$2-3$ feet

4-5 feet

B \& B

30.00

\section{PODOCARPUS}

P. macrophylla (Yew Podocarpus)

3-4 feet

4-5 feet

$B \& B$

17.50

5-6 feet

B \& B 20.00

30.00

QUERCUS (Oak)

Q. virginiana (Live Oak)

$\begin{array}{llr}\text { 4-5 feet } & \text { B \& B } & 15.00 \\ \text { 6-7 feet } & \text { B \& B } & 20.00 \\ \text { 8-9 feet, } 4 \text { inch caliper - } & \text { B \& B } & 100.00 \\ \text { 9-10 feet, 5 inch caliper } & \text { B \& B } & 130.00\end{array}$

\section{ARBORVITATES AND OTHER CONIFERS}

THUJA (Arborvitae)

T. orientalis aurea conspicua

(Goldspire Arborvitae)

$\begin{array}{llll}2-3 \text { feet } & \text { B \& B } & 27.50 \\ 3-4 \text { feet } & \text { B \& B } & 35.00 \\ 4-5 \text { feet } & \text { B \& B } & 42.50\end{array}$

$\mathrm{T}$. orientalis aurea nana (Berckmans Golden Arborvitae)

2-3 feet

B \& B $\quad 30.00$

3-4 feet

B \& B $\quad 40.00$

T. orientalis compacta (Oriental Compact Arborvitae)
2-3 feet
B \& B 22.50

$3-4$ feet -1

$T$. orientalis globosa

1-2 feet $\mathrm{B} \& \mathrm{~B} \quad 30.00$

2-3 feet

B \& B 25.00

B \& B 30.00

T. orientalis pyramidalis (Oriental Pyramidal Arborvitae)

$\begin{array}{llll}\text { 3-4 feet } & \text { B \& B } & 17.50 \\ 4-5 \text { feet } & \text { B \& B } & 27.50 \\ 5-6 \text { feet } & \text { B \& B } & 37.50 \\ \quad \text { Rosedale } & \text { B \& B } & 17.50 \\ \text { 2-3 feet } & \text { B \& B } & 30.00\end{array}$


CEDRUS

C. deodara (Himalayan Cedar)

3-4 feet 4

CHAMAECYPARIS (RETINOSPORA)

C. squarrosa veitchi (Veitch Silver Cypress)

C. pisifera plumosa

C. pisifera plumosa aurea

$\begin{array}{lll}2-3 \text { feet } & \text { B \& B } & 25.00 \\ 3-4 \text { feet } & \text { B \& B } & 35.00 \\ 4-5 \text { feet } & \text { B \& B } & 45.00\end{array}$

CUPRESSUS (Cypress) C. sempervirens horizontalis (Spreading Italian

$\begin{array}{lll}2-3 \text { feet } & \text { B \& B } & 15.00 \\ 3-4 \text { feet } & \text { B \& B } & 22.50 \\ 5-6 \text { feet } & \text { B \& B } & 35.00 \\ 6-7 \text { feet } & 50.00\end{array}$

C. sempervirens stricta (Columnar Italian Cypress)

$\begin{array}{lll}2-3 \text { feet } & \text { B \& B } & 17.50 \\ 3-4 \text { feet } & \text { B \& B } & 25.00 \\ 5-6 \text { feet } & \text { B \& B } & 45.00 \\ 6-7 \text { feet } & 60.00\end{array}$

JUNIPERUS (Juniper)

$\begin{array}{lll}\text { J. chinensis } & \text { B \& B } & 25.00 \\ 2-3 \text { feet } & \text { B \& B } & 35.00 \\ 3-4 \text { feet } & \end{array}$

J. chinensis pfitzeriana (Pfitzer Chinese Juniper)

2-3 feet spread ___ B \& B 25.00

3-4 feet spread

J. japonica sylvestris

2-3 feet _- B \& B 25.00

$3-4$ feet _ _ _ _ _ _ B B 35.00

J. Hill's silver pyramidal

3-4 feet _ B \& B 35.00

$4-5$ feet B \& B 50.00

J. virginiana (Redcedar)

3-4 feet _. B \& B 14.00

4-5 feet _ B B B 20.00

$5-6$ feet

J. virginiana cannarti (Cannart Redcedar)
$2-3$ feet

$\begin{array}{llll}2-3 & \text { feet } \\ 3-4 & \text { feet } & \text { B \& B } & 25.00 \\ 4 & & 35.00\end{array}$

4-5 feet B \& B 50.00

J. virginiana glauca (Silver Redcedar)
2-3 feet B \& $\quad 25.00$

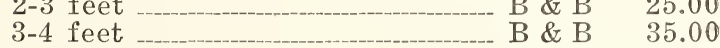

$4-5$ feet

\section{DEDCIDUOUS VINES AND CLIMBERS}

\section{BIGNONIA (Trumpetcreeper)}

B. chinensis (Red)

1 year

2 year

NB $\quad 5.00$

$\mathrm{NB} \quad 7.50$

DIOSCOREA (Yam)

D. bulbifera (Airpotato)

Strong plants

NB $\quad 5.00$

LONICERA (Honeysuckle)

L. americanna

L. sempervirens superba

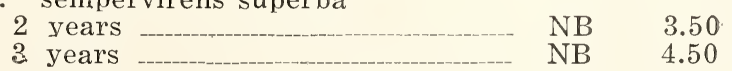

WISTERIA

W. sinensis (Chinese Wisteria)

1. year

NB $\quad 5.00$

2 year

3 year

NB $\quad 6.50$ 


\section{EVERGREEN VINES AND CLIMBERS}

\section{SIZE}

Class Per 10

BIGNONIA (Trumpetcreeper)

B. speciosa (Painted Trumpet) (Pink)

B. unguis-cati (Catlaw Trumpet) (Yellow)
1 year
NB
2 year
7.50

\section{EUONYMUS}

\section{E. radicans}

E. minima 12-15 inches 15-18 inches

FICUS (Fig)

F. pumila (repens) (Climbing Fig')

Strong potted plants

HEDERA (Ivy)

H. helix (English Ivy)

3 inch pots

5 inch pots

2 years, very heavy

NB $\quad 10.00$

TRACHELOSPERMUM

T. jasminoides (Confederate Jasmine)

Strong potted plants

\section{PALMS AND CYCADS}

\section{CURCULEGO}

C. recurvata (Weevle Palm)

5 inch pots

LIVISTONA

L. chinensis (Chinese Fan Palm)

$\begin{array}{lll}1-11 / 2 \text { feet } & \text { B \& B } & 17.50 \\ 2-21 / 2 \text { feet } & \text { B \& B } & 25.00 \\ 21 / 2-3 \text { feet } & \text { B B } & 40.00\end{array}$

PHOENIX

P. canariensis (Canary Date Palm)

$4-5$ feet

Tubs $\quad 70.00$

Tubs 80.00

WASHINGTONIA

W. robusta (California Fan Palm)

3 feet

6 feet

B \& B $\quad 30.00$

B \& B

8 feet $\quad B$ B B

50.00

100.00

SABAL

S. glabra (adansoni) (Bluestem Palmetto)

$\begin{array}{llr}\text { 4-5 feet } & \text { B \& B } & 70.00 \\ 5-6 \text { feet } & \text { B \& B } & 80.00 \\ 6-8 \text { feet } & \text { B B } & 100.00\end{array}$

TRACHYCARPUS

T. excelsa (Windmill Palm)
$21 / 2-3$ feet
$31 / 2-4$ feet
$41 / 2-5$ feet
$\mathrm{B} \& \mathrm{~B}$
$\mathrm{B} \& \mathrm{~B}$
B \& B
$\mathrm{B} \& \mathrm{~B}$
20.00
40.00
70.00
5-6 feet
100.00

\section{BAMBUSA (BAMBOO) CLUMP OR BUNCH BAMBUSA}

\section{BAMBUSA}

B. alphonsei (Alphonse Karri Bamboo)

B. argentea (Silver Bamboo)

B. argentea striata (Silver Striped Bamboo)

B. nana (Curly Bamboo)

Small clumps

B \& B $\quad 40.00$

Medium clumps

B \& B 60.00

Large clumps B \& B 100.00 


\section{BAMBUSA (BAMBOO) \\ SINGLE CANE BAMBOO}

SIZE

Class Per 10

ARUNDINARIA

A. macrosperma (Southern Cane)

A. japonica

\section{ARUNDO}

A. donax variegata (Striped Giant Reed)

\section{PHYLLOSTACHYS}

P. aurea

P. mitis (pubescens (Moso Bamboo-Edable)

P. bambusoides (Japanese Timber Bamboo) Strong canes

2-3 strong canes B \& B 10.00
B B \& B 17.50

\section{PHYLLOSTACHYS}

P. henryi (Edable Bamboo)

P. henonis

P. heteracycla

Strong canes

$2-3$ strong canes

\section{GRASSES}

BOCCONIA

C. cordata (Plumepoppy)

CYPERUS

C. papyrus (Papyrus)

\section{MISCANTHUS}

M. sinensis gracillimus (Maiden Grass)

M. sinensis variegatus (Striped Eulalia Grass)

M. sinensis zebrinus (Zebra Grass)

\section{THALIA}

T. divaricata (Bog Plant)

\section{CYNBOPOGON}

C. citratus (Lemon Grass-furnishes Oil of Citronella)

\section{SACCHARUM}

S. officinarum (Sugarcane)

\section{VITIVERIA}

V. zizanoides (Vetivere)

Small divisions

Medium clumps

Large clumps

NB

$\mathrm{NB} \quad 5.00$

NB $\quad 12.50$

\section{CYPERUS}

C. alternifolius (Umbrella-sedge)

Small divisions

Medium clumps

NB $\quad 5.00$

Large clumps

OPHIOPOGON (Snakebeard)

O. japonicus (Japanese Snakebeard)

Small divisions

Medium clumps

Large clumps

\section{PENNISETUM}

P. ruppeli (Fountain Grass)

$\mathrm{P}$. atrosanguineum

P. villosum

Small clumps

Medium clumps

Large clumps 
YUCCA (Yucca)

\begin{tabular}{|c|c|c|c|}
\hline & olia (Spanish- & & \\
\hline & years & & \\
\hline & years & & \\
\hline & aloifolia marginata & & \\
\hline & years & & \\
\hline & yea & & \\
\hline & yes & & \\
\hline & $\begin{array}{r}\text { filamen } \\
\text { or B }\end{array}$ & Adam's & \\
\hline & year di & NB & \\
\hline & $\mathrm{y}$ & $1 \sqrt{1}$ & \\
\hline & & NB & \\
\hline & ${ }_{0}^{0}$ & & \\
\hline & re & & \\
\hline & $r$ & & \\
\hline
\end{tabular}

\section{BULBS AND PLANTS}

\section{ACALYPHA}

A. hispida (Chenille Plant)

A. wilkesiana

A. wilkesiana macafeana

A. wilkesiana marginata

Rooted plants in pots

ALPINIA

A. mutica (Gingerlily)

Clumps

ASPIDISTRA

A. lurida (Common Aspidistra)

Per leaf, each $15 \mathrm{c}$

XANTHOSOMA

$\mathrm{X}$. bataviensis (Purple stem Elephants-ear) Large bulbs

COLOCASIA

C. antiquorum (Elephants-ear)

Large bulbs

NB $\quad 4.00$

C. odorata (Tree Elephants-ear)

Large bulbs

CRINUM

C. fimbriatulum (Milk-and-wine Lily) Bulbs

HEDYCHIUM (Gingerlily)

G. coronarium (Common Gingerlily)

Large bulbs

HYMENOCALLIS (Day lily)

H. fulva

H. flava

Plants

IMPATIENS

I. holstii

Rooted plants in pots

IRIS (Iris)

I. germanica (German Iris)

I. kaempferi (Japanese Iris)

2 year old plants

JACOBINIA

J. coccinea

Strong plants

LANTANA

L. camarra (various colors)

Strong potted plants 
MUSA (Banana)

M. chinensis (Finger Banana)

M. oranocensis (Oranoco Banana)

Small divisions

Medium clumps

7.50

Large clumps

SALVIA

S. leucantha (Purple Sage)

S. farinacea (Blue Sage)

Medium clumps

\title{
CITRUS FRUITS
}

\author{
ORANGE \\ JUNGLE GARDENS' HYBRID \\ (Louisiana Sweet $\mathrm{x}$ Washington Navel) \\ Bearing trees \\ GRAPE FRUIT \\ pernambuco \\ Duncan \\ Bearing trees \\ KUMQUAT \\ Round Fruited \\ Oblong Fruited \\ Trained specimens in tubs \\ Small plants in pots

\section{LEMON} \\ ponderosa \\ Bearing trees \\ 25.00
}


Acacia

Acalypha _-_ 12

Acer

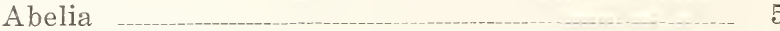

Adam's Needle, see Yucca _____ 12

Ailanthus _______________ 2

Airpotato, see Dioscorea ___ 9

Albizzia _-___- 2

Aleurites -_-_-_-_- 2

Alpinia ______________________ 12

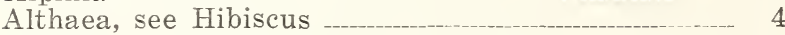

Aralia _-___-___-_ 4

Arborvitae, see Thuja ___ 8

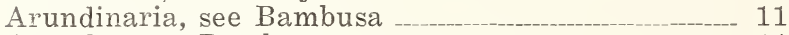

Arundo, see Bambusa _________________________________________ 11

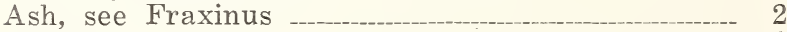

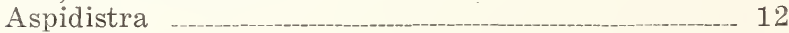

Azalia _ _ _ 5

Baccharis _______________________________________ 12

Banana, see Musa _- 13

Banana shrub, see Michelia ________________________ 7

Bamboo, see Bambusa ________________________ \& 11

Bambusa _________________________________ \& 11

Bayberry, see Myrica

Beautyberry, see Callicarpa _-___- 4

Bignonia $9 \& 10$

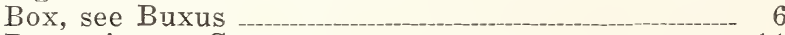

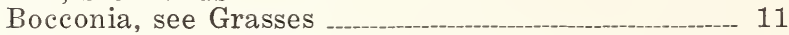

Buddleia -

Butterflybush, see Buddleia _-_- 6

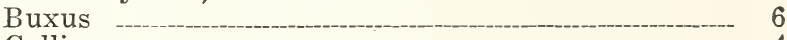

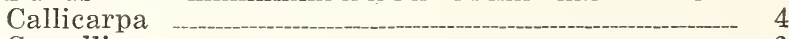

Camellia - 6

Camphor, see Cinnamomum

Cape Honeysuckle, see Tecomaria _-___- 7

Cape Jasmine, see Gardenia _.____________________________ 6

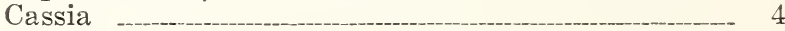

Catalpa _-_- 2

Cedrus -

Celtis -

Cercis -

Chastetree, see Vitex

Chamaecyparis

Chenille plant, see Acalypha - 12

Cherry Laurel, see Prunus

Cinnamomum

Citrus 13

Cocculus - 6

Colocasia _- 12

Confederate Jasmine, see Trachelospermum ---- 10

Cornus _-____- 2

Cyperus, see Grasses 11

Crape Míyrtle, see Lagerstroemia

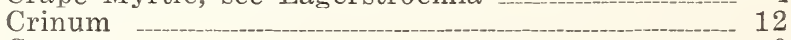

Cupressus - 12

Curculego - - 10

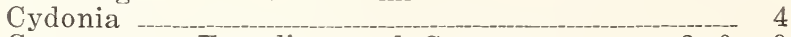

Cypress, see Taxodium and Cypressus -_-_ 3 \& 9

Cynbopogon, see Grasses _-___ 11

Daylily, see Hymenocallis --_- 12

Deutzia ___

Diervilla, see Weigelia _-_- 5

Dioscorea _- 9

$\begin{array}{ll}\text { Diospyros } & 4 \\ \text { Dogwood, see Cornus } & \end{array}$

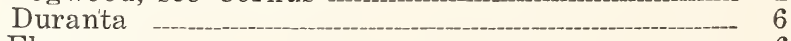

Elaeagnus - 6

Elder, see Sambucus - 3

Elephant's Ear, see Colocasia

Elm, see Ulmus -

Eriobotrya - 8 
Erythrina _-_____-

Escallonia -

Evonymus ____________ 10

Ficus

Fig, see Ficus _ 10

Forsythia

Fraxinus - 2

Gardenia _.___ 6

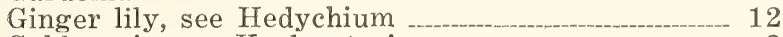

Goldenrain, see Koelreuteria ___________________________ 2

Grapefruit, see Citrus _ 13

Grasses 11

Hackberry, see Celtis _.__ 2

Hedera 10

Hedychium __________________ 12

Hibiscus

Himilayan Cedar, see Cedrus _________________________ 9

Holly, see Ilex $6 \& 8$

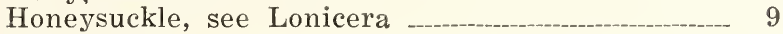

Hydrangea

Hymenocallis _-_ 12

Hypericum _-_____________ 4

Ilex

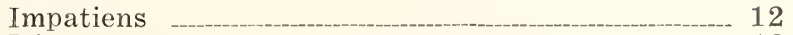

Iris - 12

Ivy, see Hedera

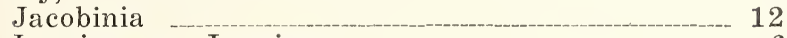

Jasmine, see Jasminum

Jasminum ____________ 6

Judas tree, see Cercis ________________________ 2

Juniper, see Juniperus ___

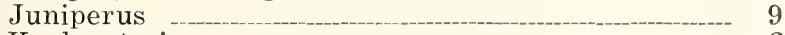

Koelreuteria

Kumquat, see Citrus 13

Lagerstroemia

Lantana

Laurustinus, see Viburnum ___

Lemon, see Citrus _- 13

Liquidambar

Ligustrum _ $\quad 6 \quad \& \quad 7$

Livistona 10

Locust, see Robinia - 3

Lonicera

Loquat, see Eriobotraya

Louisiana Sweet Bay 8

Magnolia

Magnolia fuscata, see Michelia

Malvaviscus _-___ 7

Manihot _ 2

Maple, see Acer _-_______ 2

Michelia _- 7

Melia

Milk and Wine Lily, see Crinum 12

Mimosa, see Albizzia

Miscanthus, see Grasses 11

Mombin, see Spondias

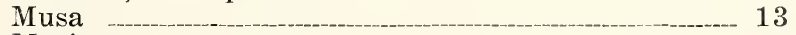

Myrica -

Myrtle, see Myrtus _-_ 7

Myrtus - 7

Nandina - -

Nerium - 7

Nyssa -

Oak. see Quercus _-___ 3

Oleander, see Nerium

Ophiopogon, see Grasses 11

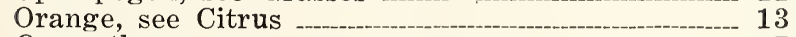

Osmanthus

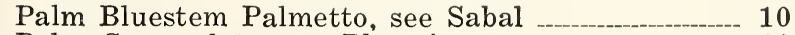

Palm Canarydate, see Phoenix 10

Palm Chinese Fan, see Livistona 10

Palm California Fan, see Washingtonia

Palm Weevle, see Curculego 10

Palm Windmill, see Trachycarpus _ 10 
Papyrus, see Cyperus

11

Parkinsonia

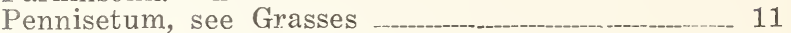

Persimmon, see Diospyros ___________________________________ 4

Phoenix - 10

Photinila

Phyllostachys, see Bambusa - 11

Pigeonberry, see Callicarpa _-______________-_ 4

Pittosporum

Plumbago _-_____- 5

Plume Poppy, see Bocconia _-_ 11

Podocarpus ___ 8

Pomegranate, see Punica _______________________ 5

Punica

Poplar, see Populus_______-__- 3

Populus ___ 3

Privet, see Ligustrum 6 \& 7

Prunus _-_- 7

Quercus $3 \& 8$

Quince, see Cydonia _____________________-_ 4

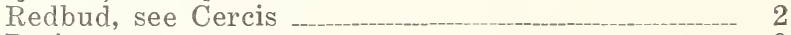

Retinospora - 9

Rhus _-_ 5

Robinia - 3

Rose Mallow, see Hibiscus _-_- 4

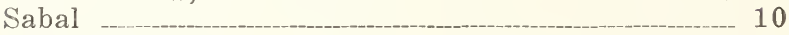

Saccharum - 11

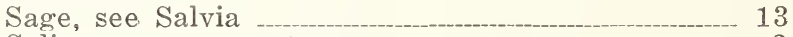

Salix

Salt Cedar, see Tamarix - 5

Salvia - 13

Sambucus - 3

Sapindus -

Sapium - 3

Sassafras - 3

Soap Tree, see Sapindus 3

Spanish Dagger, see Yucca

Spiraea _-_

Spondias - 3

St. John's Wort, see Hypericum -_- 4

Sumac, see Rhus

Sweet Gum, see Liquidambar -

Sweet Olive, see Osmanthus ________-__-_-_-_ 7

Yellow Tree, see Sapium _-_-_-_-_-_-_-_-_-_-_-_-_-_-_-_

Tamarix _ - 5

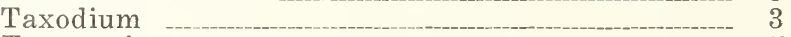

Tecomaria - 7

Texas Umbrella, see Melia _-________________-_-_-_-_ 2

Trachelospermum 10

Trachycarpus - - 10

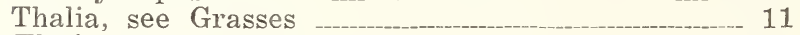

Thuja

Tree of Heaven, see Ailanthus _-___________-_ 2

Trumpet Creeper, see Bignonia - 9 \& 10

Tung, Oil Tree, see Aleurites _-___- 2

Turk's Cap, see Malvaviscus _-_-_-_- 7

Tupelo, see Nyssa _-__- 2

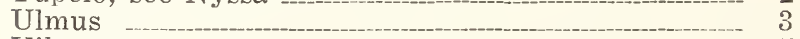

Viburnum -

Vitex - 5

Vitiveria, see Grasses 11

Washingtonia - 10

Wax Myrtle, see Myrica _-_____-_ 7

Weigelia

Willow, see Salix _-_ 3

Wisteria - 9

Xanthosoma 12

Yam, see Dioscorea - 9

Yaupon, see Ilex 6

Yew, see Podocarpus _-_ 8

Yucca 12

Yuquilla Tree, see Manihot _-_- 2 

J U N G L E G A R D E N S I N G ORPOR A T E D

Avery Island, : - : Louisiana, 